Société d'histoire de la révolution de 1848 et des

révolutions du XIXe siècle

$41 \mid 2010$

L'Algérie au XIXe siècle

\title{
Propriété, polygamie et statut personnel en Algérie coloniale, $1830-1873$
}

Property, Polygamy, and Personal Status Law in French Algeria, 1830-1873

Eigentum, Polygamie und persönlicher Status im kolonialen Algerien, 1830-1873

\section{Judith Surkis}

\section{(2) OpenEdition}

\section{Journals}

Édition électronique

URL : http://journals.openedition.org/rh19/4041

DOI : $10.4000 /$ rh 19.4041

ISSN : 1777-5329

Éditeur

La Société de 1848

Édition imprimée

Date de publication : 15 décembre 2010

Pagination : 27-48

ISSN : 1265-1354

Référence électronique

Judith Surkis, «Propriété, polygamie et statut personnel en Algérie coloniale, 1830-1873», Revue d'histoire du XIXe siècle [En ligne], 41 | 2010, mis en ligne le 30 décembre 2013, consulté le 23 mars 2021. URL : http://journals.openedition.org/rh19/4041 ; DOI : https://doi.org/10.4000/rh19.4041 


\section{JUDITH SURKIS}

\section{Propriété, polygamie et statut personnel en Algérie coloniale, 1830-1873}

Tournant dans l'histoire de l'Algérie coloniale et dans l'histoire du droit français, le sénatus-consulte de 1865 instaure une séparation entre nationalité et citoyenneté en Algérie en distinguant les citoyens des sujets "indigènes", et devient le socle d'un système juridique discriminatoire en Algérie française et dans le reste de l'empire. Cette différenciation s'appuie sur les différences entre droit civil français d'une part et droits coranique, coutumier et mosaïque de l'autre. Pour les promoteurs du texte, on trouve, au fondement de l'incompatibilité entre les systèmes juridiques, le «droit» à la polygamie et à la répudiation auxquels les sujets indigènes doivent renoncer pour devenir citoyens. Comme l'a montré Jean-Robert Henry, ce décret est marqué par l'ambiguïté puisqu'il a mis en place un régime formalisant la différence entre Français et indigènes alors même qu'il énumérait les conditions de leur assimilation juridique ${ }^{1}$.

Au moment de défendre le projet de loi devant le Sénat, le conseiller d'État Flandin affirmait ainsi que «le plein exercice des droits de citoyen français est incompatible avec la conservation du statut musulman et de ses dispositions contraires à nos lois et à nos mœurs sur le mariage, sur la répudiation, le divorce, l'état civil des enfants $»^{2}$. Le sénateur Claude Alphonse Delangle insistait également dans son rapport sur ce point, selon lui, crucial : "Ainsi la religion musulmane autorise la polygamie, la répudiation, le divorce. Il en est de même de la religion juive. Il est bien entendu que l'exer-

1. Jean-Robert Henry, «La norme et l'imaginaire, construction de l'altérité juridique en droit colonial algérien", Le procès, n 18,1987, p. 13-27. On se reportera aussi à Michael Brett, 'Legislating for Inequality in Algérie: The Senatus-Consulte of 14 July 1865', Bulletin of the School of Oriental and African Studies 51, $\mathrm{n}^{\circ}$ 3, 1988, p. 440-461; Laure Blévis, "Les avatars de la citoyenneté en Algérie coloniale ou les paradoxes d'une catégorisation", Droit et société, n 48, 2001, p. 557-580; Emmanuelle Saada, Les enfants de la colonie : les métis de l'empire français entre sujétion et citoyenneté, Paris, La Découverte, 2007; Louis-Augustin Barrière, Le statut personnel des musulmans d'Algérie de 1834 à 1962, Dijon, Editions universitaires de Dijon, 1993; Isabelle Merle, «Retour sur le régime de l'indigénat : genèse et contradictions des principes répressifs dans l'empire français", French Politics, Culture, and Society 20, no. 2, 2002, p. 77-97; Todd Shepard, The Invention of Decolonisation: The Algerian War and the Remaking of France, Ithaca, Cornell University Press, 2006.

2. Louis-Hugues Flandin dans Robert Estoublon et Adolphe Lefébure, Code de l'Algérie (18301895), tome 1, Alger, A. Jourdan, 1896, p. 303. 
cice de tels droits sera interdit à l'indigène devenu citoyen français " ${ }^{3}$. Dans cette perspective, citoyenneté française, respect du droit français et mariage monogame et indissoluble étaient intimement liés.

Il existait certes un précédent : le texte sanctionné par le Grand Sanhédrin de 1807 faisait de la renonciation à la polygamie et à la répudiation une condition de l'assimilation des Juifs à la nation française et de leur accession à la citoyenneté . Quelques décennies plus tard, les réformateurs qui cherchaient à "régénérer» les Juifs d'Algérie en faisant d'eux des citoyens français prirent l'expérience napoléonienne comme modèle. La morale et les coutumes familiales seraient ainsi devenues, comme l'a suggéré Joshua Schreier, les composantes essentielles d'une «mission civilisatrice» visant les juifs ${ }^{5}$. Le lien entre le statut de droit civil et la citoyenneté, cimenté par le sénatus-consulte de 1865, n'est cependant pas systématique : les sujets coloniaux des quatre communes du Sénégal et des cinq établissements français de l'Inde s'étaient ainsi vus accorder la citoyenneté en 1848 alors qu'ils conservaient un statut distinct en matière de droit civil ${ }^{6}$.

Pourquoi dès lors la différence de statut civil, et, plus particulièrement, le «droit» à la polygamie, furent-ils érigés en obstacle à la citoyenneté française dans le droit colonial algérien et comme fondement de la sujétion des indigènes? Comme bien d'autres questions centrales de l'histoire de l'Algérie au XIX ${ }^{\mathrm{e}}$ siècle, celle-ci ne saurait être traitée sans prendre en compte les débats sur les droits fonciers des indigènes, eux-mêmes associés aux différentes manières d'envisager la "loi musulmane» et aux différentes options en matière de colonisation. Le destin juridique de la polygamie dans l'Algérie du XIX ${ }^{\mathrm{e}}$ siècle ne renvoie pas seulement à l'articulation entre moralité et citoyenneté. Son histoire est aussi liée à la dissociation progressive du droit foncier d'un «droit familial» musulman. En effet, parallèlement au sénatusconsulte de 1865, un ensemble de nouvelles lois contribuèrent à créer et distinguer un «statut réel» modifiable d'un «statut personnel» musulman supposé inaltérable . p. 305

3. Rapport Delangle, cité dans Robert Estoublon et Adolphe Lefébure, Code de l'Algérie, op. cit.,

4. Sur ce point, on se reportera à Simon Schwarzfuchs, Napoleon, the Jews, and the Grand Sanhedrin, Londres, Routledge, Kegan, and Paul, 1979; Paula Hyman, The Jews of Modern France, Berkeley, University of California Press, 1998; Ronald Schechter, Obstinate Hebrews: Representations of Jews in France, 1715-1815, Berkeley, University of California Press, 2003.

5. Joshua Schreier, 'Napoléon's Long Shadow: Morality, Civilization and Juifs in France and Algérie, 1808-1870', French Historical Studies, tome 30, n 1, 2007, p. 77-103.

6. Sur ce point, voir Patrick Weil, Qu'est-ce qu'un Français? Histoire de la nationalité française depuis la Révolution, Paris, Grasset, 2002, p. 235. Plusieurs mesures viennent cependant limiter l'exercice de ces droits, $c f$. Damien Deschamps, «Une citoyennete différée : sens civique et assimilation des indigènes dans les Etablissements français de l'Inde", Revue française de science politique 47, n 1, 1997, p. 49-69; Catherine Coquery-Vidrovitch, «Nationalité et citoyenneté en Afrique occidentale française : Originaires et citoyens dans le Sénégal colonial ", Journal of African History, tome 42, n 2, 2001, p. 285-305.

7. On distingue en droit le «statut personnel» (ensemble des lois qui régissent le statut civil des personnes physiques) du «statut réel» qui s'applique aux choses. 
Cet article se penche sur la généalogie de cette dissociation pour mettre en lumière les causes et les modalités selon lesquelles la moralité familiale est devenue centrale dans le statut juridique différent des Algériens. Ainsi, plutôt que de postuler que le "droit personnel» est un domaine spécifique - et spécifiquement problématique - du «droit musulman», il s'agit ici de montrer comment ce domaine s'est constitué ${ }^{8}$. Les trajectoires contemporaines du droit du statut personnel «israélite» et les débats sur l'extension du droit foncier français aux musulmans expliquent comment la polygamie est devenue une composante essentielle de cette construction.

Les autorités coloniales n'ont pas toujours vu la polygamie indigène comme un problème essentiellement religieux ou juridique. Dans les premières décennies de la conquête, la "polygamie» est souvent décrite par les militaires et les colons comme une institution liée à l'économie, qui pourrait être réformée par des politiques appropriées, menées par les autorités militaires ou civiles. La définition plus tardive de la polygamie comme «droit» coranique irréductible - et intolérable - fut intimement liée à des débats politiques houleux sur la colonisation et, notamment, sur la place des indigènes algériens dans l'ordre colonial. Dans cet article, je propose de rendre visibles les divers éléments, parfois contradictoires, à partir desquels s'est façonnée cette image de la différence juridique algérienne musulmane, aussi tenace que politiquement utile, sans être pour autant stable ou cohérente. Cette généalogie de la polygamie algérienne montre comment la différence, sur des questions familiales, entre le droit "français" et le droit «indigène " est devenue un pilier de l'ordre colonial algérien.

\section{UNE FICTION PERSISTANTE}

L'Algérie est associée à la "polygamie» depuis Montesquieu, qui présentait l'excès de sensualité à la fois comme une cause et comme un symptôme de despotisme, et, in fine, de décadence' ${ }^{9}$. Ces associations perdurent après la conquête d'Alger en 1830. Lors des premières années de la colonisation, des peintres romantiques comme Eugène Delacroix et Théodore Chassériau mettent en scène des harems mythiques peuplés de femmes alanguies aux pauses langoureuses. On a pu voir dans ces images des fictions niant la violence de la conquête ${ }^{10}$.

8. Mon approche differe ici de celle des historiens du droit qui voient le «statut personnel» comme une notion immuable. $C f$. Louis-Augustin Barrière, Le statut personnel des musulmans d'Algérie de 1834 à 1962, op. cit.

9. Montesquieu, Euvres complètes, texte annoté et présenté par Roger Caillois, Paris, Gallimard, Bibliothèque de la Pléiade, tome 1, 1989, Livre XVI, Chapitre VI ("De la polygamie en elle-même»), p. 512-513. Cf. Alain Grosrichard, La structure du sérail : la fiction du despotisme asiatique dans l'Occident classique, Paris, Seuil, 1979.

10. Darcy Grimaldo Grigsby, 'Orients and Colonies: Delacroix’s Algérien Harem', in Beth S. Wright [dir.], The Cambridge Companion to Delacroix, Cambridge, Cambridge University Press, 2001, p. 69-87 
Un article sur «les danses maures" publié dans La Revue africaine en 1837 reprend ce fantasme. Après avoir cité les tableaux de Delacroix, l'auteur s'abandonne à une rêverie où des danseuses rencontrées au bordel d'une ville conquise lui apparaissent comme les habitantes d'un harem interdit ${ }^{11}$. Fasciné par ce spectacle, il s'enthousiasme pour l'organisation «musulmane» de la différence sexuelle : "Au harem! Au harem! Là du moins vous ne nous échapperez pas; votre corps, votre âme, nous les enfermerons dans une étroite prison ${ }^{12}$. L'encadrement récent de la prostitution par les Français et le phénomène émergent du tourisme colonial auraient favorisé de tels fantasmes ${ }^{13}$.

La condamnation morale constitue l'envers de ces fictions érotiques. Dans la continuité de Montesquieu, la polygamie est associée au despotisme; elle est également tenue pour nocive pour la santé, suivant l'opinion de la plupart des médecins de l'époque ${ }^{14}$. Ces dénonciations rejoignent les critiques religieuses de la polygamie, telles celles de Louis de Bonald ${ }^{15}$. On lit ainsi, sous la plume des collaborateurs de la Revue de l'Orient, dans le cadre d'une entreprise de soutien aux intérêts catholiques en "Orient", un catalogue des effets négatifs de l'Islam sur la "civilisation orientale». Comme les mœurs familiales sont au cœur de sa vision politico-religieuse, le journal voit dans la polygamie " un obstacle à la civilisation $»^{16}$.

Depuis les travaux d'Edward Saïd, ces représentations d'un "Orient» décadent, dévirilisé et désordonné apparaissent comme des éléments de l'affirmation d'une supériorité culturelle européenne. Ceux qui tentent de pérenniser la domination française en Algérie au début des années 1830 ne placent pas pour autant ces fantasmes au cœur de leurs analyses ${ }^{17}$. Quand bien même leurs écrits diffusent certains stéréotypes, les militaires - et notamment les

et 'The Women of Algiers', in Todd Porterfield (ed.), The Allure of Empire: Art in the Service of French Imperialism, 1798-1836, Princeton, Princeton University Press, 1998, p. 117-41.

11. Ch. de L., "Danses maures - Opinion sur les harems", Revue africaine, n 5, 1837, p. 64.

12. Ibidem, p 68.

13. Edouard Adolphe Duchesne, De la prostitution dans la ville d'Alger, Paris, J.-B. Ballière, 1853, p. 87-90. Cf. Marnia Lazreg, The Eloquence of Silence: Algerian Women in Question, Londres, Routledge, 1994, p. 29-33; Christelle Taraud, La prostitution coloniale : Algérie, Tunisie, Maroc (1830-1962), Paris, Payot, 2003.

14. Cf. Jérôme Adolphe Blanqui, «La polygamie en Orient", in Considérations sur l'état social de la Turquie d'Europe, Paris, W. Coquebert, 1842 p. 67-77; Eusèbe de Salle, «Mémoire sur la polygamie musulmane", Journal des économistes, tome 3, 1842, p. 171-187. Sur les aspects médicaux, $c f$. Julien Joseph Virey, Hygiène philosophique appliquée à la politique et à la morale, Paris, Librairie de Crochard, 1831 ; Lallemand, "De l'Orient et de l'Occident : Considérations physiologiques ", Revue du Midi, tome $2, \mathrm{n}^{\circ} 1,1843$, p. $1-20$.

15. Louis de Bonald, Euvres complètes, tome 2, Paris, J.-P. Migne, 1863, p. 105.

16. "Quelle est l'influence de la religion musulmane sur la civlisation des pays de l'Orient ou domine l'Islamisme?», Revue de l'Orient, 1, 1843, 222. Cf. Fortin d'Ivry, "Orient et Occident», Revue de l'Orient, 4, 1844, tome 4, p. 211 ; Lamouroux, "De la polygamie en Algérie", Revue de l'Orient, tome 10, 1851, p. 41-44. Sur la politique religieuse en Algérie, voir Sarah A. Curtis, 'Emilie de Vialar and the Religious Reconquest of Algérie', French Historical Studies, vol. 29, n 2, 2006, p. 261-292.

17. Sur la distinction entre représentation coloniale et empirisme de la loi coloniale, Ann Laura Stoler, Carnal Knowledge and Imperial Power: Race and the Intimate in Colonial Rule, Berkeley, University of California Press, 2002, p. 44. 
chefs de bureaux arabes - dans leur pratique de gouvernement des tribus nouvellement assujetties, cherchent avant tout à minimiser l'importance des différences culturelles.

\section{Des sujets semblables}

Les premiers partisans - surtout militaires - d'une «fusion» des populations, minimisent les différences sexuelles et religieuses entre Français et indigènes ${ }^{18}$. Le général Franciade-Fleurus Duvivier, par exemple, va jusqu’à établir des parallèles entre le droit familial musulman et celui des Français. À son retour en métropole, il fait campagne en faveur de la colonisation dans un traité largement diffusé, La solution de la question d'Algérie. Tout en défendant le rôle de l'armée, il critique vivement ceux qui exagèrent le statut inférieur des femmes "arabes ${ }^{19}$. Considérant leur condition comme une question économique et sociale, il affirme : «Ce n'est pas la dureté de cœur, ce n'est pas surtout la religion, qui sont causes de ces différences; c'est la force des choses qui les commande impérieusement ${ }^{20}$. Il avance même, dans un parallèle audacieux, que le code civil, comme la loi islamique, oblige les femmes à être obéissantes et soumises à leur mari ${ }^{21}$. La "solution de la question d'Algérie» qu'il propose atténue donc les différences sur les plans religieux et juridique. D'autres militaires de haut rang, comme Henri Pellissier de Reynaud ou Eugène Daumas, se font l'écho de cette explication avant tout économique de la situation inférieure des femmes arabes ${ }^{22}$.

Alors que la "fusion" des populations et des lois demeure un horizon lointain, les autorités françaises maintiennent des juridictions séparées pour les affaires civiles en Algérie ${ }^{23}$. Ce principe structure l'ordonnance de 1834 sur l'"organisation judiciaire des possessions françaises du nord de l'Afrique", promulguée conjointement par les ministères de la Guerre et de la Justice. Une version préliminaire de cette ordonnance, ébauchée par le garde des Sceaux, comprenait une clause visant à limiter, au nom de l'ordre public, la

18. Cheryl Welch, 'Colonial Violence and the Rhetoric of Evasion: Tocqueville on Algérie', Political Theory, 31, n 2, 2003, p. 243.

19. En particulier Jean Jacques Baude, L'Algérie, tome 1, Paris, Arthus Bertrand, 1841.

20. Franciades-Fleurus (général) Duvivier, Solution de la question de l'Algérie, Paris, Gaultier-Laguionie, 1841 , p. 320.

21. Ibidem, p. 321-25.

22. Edmond Pellissier de Reynaud, Annales algériennes (nouvelle édition, revue, corrigée et continuée jusqu'à la chute d'Abd-el-Kadr; avec un appendice), tome 3, Paris, J. Dumaine, 1854, p. 440-41; Eugène Daumas, La femme arabe, Alger, Adolphe Jourdan, 1912, p. 141. Cf. Julia Clancy-Smith, 'La Femme Arabe: Women and Sexuality in France's North African Empire', in Amira El Azhary Sonbol [dir.], Women, the family, and divorce laws in Islamic History, Syracuse, Syracuse University Press, 1996 p. 52-63.

23. Prosper Enfantin, La colonisation de l'Algérie, Paris, P. Bertrand, 1843 et Exposé de l'état actuel de la société arabe, du gouvernement et de la législation qui la régit, Alger, Imprimerie du gouvernement, 1844. 
portée du droit algérien : "Néanmoins il ne peut être dérogé à la loi en tout ce qui intéresse l'ordre public et les bonne mœurs». On pouvait cependant estimer que cette clause serait difficilement applicable. "Et la polygamie?", note un administrateur en marge du projet, comme pour évoquer les problèmes évidents que cette proposition pouvait susciter (elle fut d'ailleurs rapidement éliminée $)^{24}$. Le ministère de la Guerre hésite alors beaucoup à ordonner trop hâtivement la souveraineté du code «français» qui "ainsi imposée, ne sera que nominale ou se rendra odieuse $»^{25}$.

Ainsi, alors que l'ordonnance du 28 février 1841 étend le code pénal français au territoire algérien, l'ordonnance du 26 septembre 1842 maintient les tribunaux islamiques pour les «musulmans» et le «statut personnel mosaïque» pour les questions de mariage, de divorce et d'héritage ${ }^{26}$. Les juifs sont soumis au code civil pour les questions de propriété («statut réel»), alors que les musulmans ne le sont pas. La juridiction des tribunaux «français» et «indigènes» évolue au cours des premières décennies de la colonisation. Une série de lois éliminent les tribunaux rabbiniques au cours des années 18401850 , et mettent en place la structure et l'organisation de tribunaux «musulmans $»^{27}$ solidifiant la distinction entre une population arabe assujettie à la loi coranique et des populations berbères soumises au «droit coutumier $»^{28}$.

L'établissement formel des bureaux arabes pour administrer la plus grande partie des territoires nouvellement conquis ne fait que renforcer la multiplicité des juridictions. En confiant le maintien de l'ordre aux élites et à la justice indigènes, les militaires adoptent une attitude pragmatique à l'égard du droit musulman. Dans certains cas, ils présentent la loi coranique non pas comme un facteur de décadence mais plutôt comme une source de renouveau social et un élément à utiliser dans le cadre de leur politique de transformation des modes de vie «nomades" des tribus rurales ${ }^{29}$.

24. Voir l'article 34 de la version annotée du projet conservé aux Archives nationales (Arch. nat.), BB30 616. Pour la version finale du 10 août 1834, cf. Bulletin des lois du Royaume de France, $2^{\mathrm{e}}$ partie, $1^{\text {re }}$ section, $\mathrm{n}^{\circ} 324,1834$.

25. AN BB30 616, Rapport, ministère de la Guerre, «Observations sur le projet d'ordonnance royale relative à l'organisation de la Justice en Afrique».

26. "Ordonnance sur l'organisation de la justice en Algérie (26 septembre 1842)», in Robert Estoublon et Adolphe Lefébure, Code de l'Algérie, op. cit., p. 22-30.

27. Cf. l'ordonnance portant organisation du culte israélite en Algérie du 9 novembre 1845, Code de l'Algérie annoté, tome 1, Alger, Louis Jourdan, 1896 (p. 82-85), le décret portant organisation de la justice musulmane du 1er octobre 1854 (Code de l'Algérie, op. cit, p. 173-178), et le décret qui organise la justice musulmane du 31 décembre 1859 (Code de l'Algérie, op. cit., p. 230-237).

28. Jean-Robert Henry, «La norme et l'imaginaire», loc. cit., p. 18. Pour les tribunaux rabbiniques, "L'ordonnance portant organisation du culte israélite en Algérie du 9 novembre 1845 », Code de l'Algérie annoté, p. 82-85; pour les tribunaux musulmans, «Décret portant organisation de la justice musulmane du $1^{\text {er }}$ octobre 1854", Code de l'Algérie, p. 173-178, et «Décret qui organise la justice musulmane du 31 décembre 1859", Code de l'Algérie, p. 230-237.

29. Voir les débats dans Michael Brett, 'Legislating for Inequality in Algérie', loc. cit. 445-6, et JeanLoup Amselle, Vers un multiculturalisme français : l'empire de la coutume, Paris, Champs Flammarion, 2001. Sur ces officiers, Patricia Lorcin, Imperial Identities: Stereotyping, Prejudice, and Race in Colonial Algérie, London, I. B. Tauris, 1999, p. 99-117. 
Les pamphlets rédigés par l'officier Charles Richard, en poste au bureau arabe d'Orléansville, illustrent bien cette tendance ${ }^{30}$. Il dénonce la polygamie comme "l'immoralité la plus honteuse», mais considère néanmoins le droit musulman comme un instrument de moralisation et de domination ${ }^{31}$. Il suggère notamment que les Français pourraient révéler à la femme musulmane les droits que lui octroie le Coran : «étonnée d'avoir des droits, elle songera à les demander tout entier, elle voudra régner seule dans la tente, et sera fort surprise d'apprendre encore de nous que la loi l'y autorise, et qu'elle peut étouffer du pied la polygamie, qui est son ennemie la plus cruelle ${ }^{32}$. Dans le modèle de gouvernement du "peuple conquis" qu'il élabore par la suite, la «féodalité» française "améliorera ses mœurs et sa moralité à l'aide d'une interprétation intelligente de la loi musulmane elle-même; elle étouffera les vieilles coutumes barbares, particulièrement la polygamie, avec les secours de cette loi; et la femme, devenue plus libre, élèvera d'autant la dignité de l'homme $»^{33}$.

Charles Richard s'oppose à l'extension du code civil aux peuples conquis d'Algérie pour des raisons pratiques. Les poursuites pour cause de bigamie, d'après lui, risqueraient de devenir un véritable fardeau pour l'administration française. Comme pour les auteurs de l'ordonnance de 1834, la polygamie apparait comme le nœud des conflits juridiques qu'entraînerait nécessairement toute tentative d'application du code civil dans la colonie ${ }^{34}$. Pour Charles Richard, cette seule menace démontre la nécessité "de laisser au peuple arabe sa loi musulmane, en la soumettant à une surveillance intelligente dans son application, et de manière à préparer les invasions successives de la nôtre ${ }^{35}$. Il résume là l'un des principaux objectifs de l'institution des bureaux arabes.

Les arguments avancés par Charles Richard expliquent que l'armée ait cherché à préserver le système judiciaire musulman dans le contexte d'une opposition toujours plus intense au décret du $1^{\text {er }}$ octobre 1854 octroyant à ces tribunaux une plus grande autonomie ${ }^{36}$. Les colons et les magistrats français, cherchant à étendre les compétences des juridictions civiles françaises, ont critiqué l'indépendance des tribunaux musulmans, dans le cadre d'une campagne plus vaste contre les bureaux arabes. Les militaires, au contraire, maintenaient que l'existence de juridictions distinctes permettait d'éviter de

30. Xavier Yacono, Les bureaux arabes et l'évolution des genres de vie indigènes dans l'ouest du Tell Algérois (Dahra, Chélif, Ouarsenis, Sersou), Paris, Larose, 1953, p. 136-45. p. 53.

31. Charles Richard, Du gouvernement arabe et de l'institution qui doit l'exercer, Alger, Bastide, 1848,

32. Ibidem, p. 56.

33. Charles Richard, De la civilisation du peuple arabe, Alger, Dubos Frères, 1850, p. 29.

34. Charles Richard, Du gouvernement arabe, op. cit., note 20, p 77.

35. Ibidem, p. 81.

36. Décret portant organisation de la justice musulmane in Robert Estoublon et Adolphe Lefébure, Code de l'Algérie, op. cit., 173-78. Cf. Allan Christelow, Musulman Law Courts and the French Colonial State in Algérie, Princeton, Princeton University Press, 1985 et Michael Brett, 'Legislating for Inequality in Algérie,' loc. cit., p. 443-45. 
nombreux conflits et faisait avancer plus rapidement l'œuvre de colonisation.

Ce débat sur la justice participe d'une controverse plus vaste sur la capacité de l'armée à coloniser. Les colons, qui voyaient dans l'administration militaire un obstacle, dénonçaient les projets de l'armée comme utopiques et accusaient le personnel des bureaux arabes de violence, de corruption et d'immoralité ${ }^{37}$. C'est en réponse à de telles attaques que Ferdinand Hugonnet, s'inspirant de son expérience personnelle à Bône, La Calle, et Constantine, dresse un portrait du "chef de Bureau arabe». D'après lui, le chef est capable de résoudre les conflits entre indigènes et de mettre en ouvre des réformes; il conseille de ne pas intervenir dans le domaine de la religion, et au-delà, de ne pas tenter de soumettre les indigènes à l'administration civile française. Face à «certaines opinions conçues trop légèrement», Ferdinand Hugonnet souligne : «la femme, devant la loi musulmane et dans la société indigène, est moins bien traitée que chez nous, je le reconnais, mais de là à admettre toutes les conclusions qui ont été exposées à ce propos, il y a loin $»^{38}$.

Il ne prône en rien un quelconque relativisme culturel et dénonce la polygamie comme «le grand vice [...] de l'état actuel de la société indigène». Il attribue cependant sa persistance non pas à la religion ni même à "des goûts sensuels ", mais à des facteurs économiques : "aussitôt qu'une tente a un peu d'aisance, il faut plusieurs femmes dans l'intérieur " ${ }^{39}$. Comme chez Duvivier ou Richard, cette interprétation économique de la polygamie engage un projet impérial bien précis. Ferdinand Hugonnet affirme en effet que les progrès matériels réalisés par les Français - comme l'introduction de moulins à grain et de boulangeries - élimineront progressivement la polygamie. De tels équipements, financés par l'entremise des bureaux arabes, peuvent en effet favoriser l'enracinement des tribus indigènes tout en faisant naittre en eux la loyauté à l'égard du gouvernement français et produire, de surcroit, cet heureux résultat : «le cultivateur commencera à s'apercevoir qu'une femme peut lui suffire ${ }^{40}$.

37. Contre les colonies militaires, Capo de Feuillide (Jean Gabriel Cappot), L’Algérie française, Paris, Plon, 1856, p. 34-41; Clément Duvernois, L’Algérie ce qu'elle est-ce quelle doit être : essai économique et politique, Bibliothèque algérienne, Alger, Dubos, 1858, p. 248. Cf. Annie Rey-Goldzeiguer, Le royaume arabe : la politique algérienne de Napoléon III, 1861-1870, Alger, Société nationale d'édition et de diffusion, 1977 , p. 113 .

38. Ferdinand Hugonnet, Souvenirs d'un chef de Bureau arabe, Paris, Michel Lévy, 1858, p. 113. Sur la carrière de Ferdinand Hugonnet, on se reportera à Patricia Lorcin, Imperial Identities, op. cit., p. 303.

39. Ferdinand Hugonnet, Souvenirs..., op. cit., p. 96.

40. Ibidem, p. 117. 


\section{LA QUESTION DU DROIT MUSULMAN}

Les colons et leurs partisans, cependant, voient la persistance de la polygamie comme un symptôme de l'échec du pouvoir militaire : la division du territoire algérien et l'existence de juridictions séparées nuiraient à l'intégration des populations indigènes. Dans l'espoir d'augmenter les surfaces à leur disposition, ils réclament la «libre» colonisation par les Européens et «l'assimilation légale», c'est-à-dire l'application uniforme d'un «droit commun». Quand Napoléon III institue un ministère de l'Algérie dirigé par des civils, les partisans des colons croient ces réformes à portée de main, mais, loin de se résorber, le conflit avec les militaires ne fait que s'exacerber ${ }^{41}$.

Personne ne défend plus ardemment la cause de la "colonisation libre» que Clément Duvernois, jeune polémiste ambitieux, auteur de nombreux pamphlets. Se présentant comme un véritable patriote, malgré son opposition à la politique menée en Algérie, il déclare par exemple que «l'Algérie est française ou elle ne l'est pas; si elle n'est pas française, nous n'y avons que faire; si elle est française, toutes les populations qui l'habitent doivent accepter nos lois, ou se retirer ${ }^{42}$. Les textes de Clément Duvernois considèrent la polygamie comme un obstacle à l'uniformité juridique de l'Algérie mais lui donnent une explication d'ordre économique, comme le font ses adversaires de l'administration militaire. "Tolérée et non pas ordonnée par le Coran", la polygamie n'est pas, insiste-t-il, un principe religieux fondamental ${ }^{13}$. Elle ne suffit donc pas à justifier une séparation entre l'autorité civile et militaire ou entre des juridictions française et musulmane ${ }^{44}$. Poussant à son extrême le libéralisme qu'il considère comme essentiel à la colonisation de l'Algérie, Clément Duvernois va jusqu'à proposer la suppression complète de l'institution du "mariage civil» en Algérie. Comme il doute cependant qu'une solution aussi radicale soit acceptée, il suggère $d$ ' " admettre provisoirement la polygamie, en prenant toutes les mesures propres à en diminuer la mise en pratique». En effet, l'augmentation de la population européenne et l'industrialisation devraient faire reculer le besoin de main d'œuvre féminine, «la cause principale» selon lui de la polygamie ${ }^{45}$.

Les magistrats français qui siègent en Algérie critiquent eux aussi l'existence de plusieurs juridictions. Pour des raisons professionnelles, leurs intérêts se rapprochent de ceux des colons. Des rapports commandés par le

41. Robert Moulis, "Le Ministère de l'Algérie (24 juin 1858-24 novembre 1860)" (Université d'Alger, 1926).

42. Clément Duvernois, L'Algérie ce qu'elle est-ce qu'elle doit être : essai économique et politique, p. 252-3.

43. Ibidem, p. 101, p. 254.

44. Clément Duvernois, L'Akhbar et les novateurs téméraires; lettre à M.A. Bourget, Alger, Dubos frères etc., 1858 , p. 11.

45. Clément Duvernois, La réorganisation de l'Algérie; lettre à S.A.I. le prince Napoléon, Alger, Dubos frères etc., 1858, p. 14-15. Voir aussi Feuillide (Jean Gabriel Cappot), L'Algérie française, p. 246-50; Dr. Cauquil, Etudes économiques sur l'Algérie, Oran, Adolphe Perrier, 1860, p. 74-76. 
ministère de l'Algérie et des Colonies sur les réformes judiciaires révèlent ainsi de nettes divergences entre militaires et civils sur la justice islamique ${ }^{46}$. Une réforme adoptée en 1859 accorde certaines concessions aux partisans du gouvernement civil ${ }^{47}$ en réinstaurant la tutelle des cours d'appel françaises sur les juridictions musulmanes, supprimée par le décret de $1854^{48}$. Ainsi placée au cœur des débats sur l'organisation judiciaire de l'Algérie, la polygamie est devenue le symbole des difficultés d'application des principes du droit français aux musulmans.

\section{Propriété ET MORALITÉ}

Après la suppression du ministère de l'Algérie en 1860, les militaires reprennent les rênes du pouvoir. Au cours des années suivantes, la politique dite du "royaume arabe» cherche à définir et délimiter les droits des indigènes sur les questions du territoire, de la nationalité et des institutions judiciaires. Napoléon III, qui effectue deux voyages en Algérie, est à l'origine des sénatus-consultes de 1863 et 1865 définissant respectivement la propriété «indigène» et les conditions d'accession des «indigènes» et des étrangers à la pleine citoyenneté.

Les colons réclament depuis longtemps l'application d'un «droit commun ». Pour l'un de leurs représentants, «il faut s'attacher seulement à séparer dans le Coran le spirituel du temporel, il faut soumettre la propriété au Code Napoléon $»^{49}$. Une large partie des colons intervenant dans le débat sont alors loin de concevoir la polygamie comme une caractéristique de la différence insurmontable que représente la religion des indigènes et comme un obstacle dirimant au projet d'assimilation juridique. Pour eux, il s'agit avant tout de promouvoir le droit foncier français, surtout après le sénatus-consulte de 1863 qui le mit à mal. Tout en redessinant les bornes des propriétés tribales, avec des effets perturbateurs évidents, le texte de 1863 promettait en effet de protéger ces mêmes terres en tant que "propriétés communes» - répertoriées sous l'appellation "arch» par les Français - et de limiter l'extension de la "propriété individuelle " ${ }^{50}$. Ces dispositions étaient fondées sur la présomption que, pour les indigènes «musulmans ", l'«indivision" était "non seulement conforme à leurs habitudes demi-nomades, mais encore à leurs

46. Arch. nat. Outre-Mer, 17 H 2, Secrétaire général au Ministre des colonies, «Résumé des rapports de Généraux et des Préfets, du Premier Président et du Procureur Général sur la réorganisation de la Justice Musulmane (1859)", cf. Allan Christelow, Musulman Law Courts, op. cit., p. 164-66; Moulis, "Le Ministère de l'Algérie», op. cit., p. 234-45.

47. "Rapport à l'empereur sur le décret qui organise la justice musulmane (31 décembre 1859)",

Robert Estoublon et Adolphe Lefébure, Code de l'Algérie, op. cit., p. 231.

48. Ibidem. Cf. Michael Brett, 'Legislating for Inequality in Algérie', loc. cit., p. 447-49.

49. Algérie : Immigrants et indigènes, Paris, Challamel, 1863, p. 22. Il s'agissait d'une réponse à Ismaël Urbain, L'Algérie française : indigènes et immigrants (1862), Paris, Séguier, 2002.

50. Cf. l'article de Didier Guignard dans la présente livraison. 
préjugés religieux " ${ }^{51}$. Le sénatus-consulte de 1863 se présente donc en partie comme respectueux du droit religieux indigène, alors que pour les colons, «la propriété individuelle sera le plus puissant moyen de civilisation de fusion des deux races et de progrès ${ }^{52}$.

Ce débat sur l'expansion de la "propriété individuelle» au territoire algérien coïncide avec celui sur la nationalité et sur l'application du droit français aux Algériens indigènes. Au cours de ces années, les conseils généraux des trois départements d'Algérie examinent fréquemment ces questions. Tout en appelant à faciliter la naturalisation des colons espagnols, italiens et maltais, ils réclament des lois permettant aux sujets "indigènes» de devenir citoyens français. Ainsi, en 1860, le président du Conseil d'Alger, Charles de Vaulx, également premier président de la Cour impériale d'Alger, formule-t-il le projet d'une naturalisation "volontaire ${ }^{53}$.

Cette possibilité d'accession individuelle à la citoyenneté représente une alternative à la naturalisation collective réclamée par certains délégués «israélites» et à laquelle s'opposent les représentants des musulmans. Comme l'explique de Vaulx, on ne saurait imposer aux indigènes musulmans "pris en masse, avec la qualité de Français, des obligations qui pourraient être aussi mal comprises que peu respectées $»^{54}$. Inquiets de voir à nouveau réduits les droits attachés à leur statut civil, et notamment sur les questions du mariage, de la séparation, du divorce et des successions, les deux délégués musulmans, Ahmed-Boukandoura et Hassan-ould-Caïd-Ahmed, souscrivent à ces arguments : la naturalisation, même volontaire, risquerait de porter atteinte, selon eux, au droit «religieux» musulman. Un de ces représentants, identifié dans les procès-verbaux sous le simple nom $d$ '«indigène», explique ainsi que «le musulman qui se fait naturaliser doit accepter le code civil, et, par suite, se trouve obligé de renoncer à notre loi, qui est, avant tout, essentiellement religieuse ${ }^{55}$. Cette loi "religieuse», qui règle les questions de mariage, de divorce et d'héritage, apparaît dès lors comme un obstacle majeur à l'accession à la citoyenneté. À propos de la polygamie, ce même représentant note que si les «Maures», qui habitent les villes, sont bien monogames, il n'est pas rare de voir «l'Arabe nomade qui vit sous la tente [...] en possession de trois à quatre femmes. Comment le décider à abandonner une condition que la loi ne prohibe pas, et qui, d'ailleurs, rentre si bien dans les mœurs du pays qu'il

51. «Rapport fait au nom de la commission du Sénat, par M. de Casabianca, 8 avril 1863», sur le "Sénatus-consulte relatif à la constitution de la propriété en Algérie, dans les territoires occupés par les Arabes", in Robert Estoublon et Adolphe Lefébure, Code de l'Algérie, p. 273. Sur l'évolution du droit de propriété, on se reportera à Jean Louis Autin, «La législation foncière en Algérie de 1830 à 1870 ou le triomphe de la raison juridique coloniale», Le procès, $n^{\circ} 18,1987$, p. $85-97$

52. "Rapport fait au nom de la commission du Sénat, par M. de Casabianca, 8 avril 1863 ", in Robert Estoublon et Adolphe Lefébure, Code de l'Algérie, p. 274.

53. Séance du 9 octobre 1860, Conseil général d'Alger, Procès-verbaux, 1860, p. 120. Cf. Conseil général d'Oran, Procès-verbaux, 1859, p. 170-174.

54. Conseil général d'Alger, Procès-verbaux, 1860, p. 105.

55. Séance du 9 octobre 1860, Conseil général d'Alger, Procès-verbaux, 1860, p. 115-6. 
habite?» Il conclut alors que "nos mœurs et notre loi s'opposent à la naturalisation». C'est donc en évoquant les liaisons polygames de l'Arabe nomade que ce représentant "musulman" explique son refus de la naturalisation et des droits politiques afférents ${ }^{56}$.

Quoi qu'il en soit, juifs et musulmans sont en situation minoritaire au Conseil. Les colons dénoncent l'absence de libéralisme de leurs positions et adoptent une résolution favorable à la naturalisation "individuelle" par un vote de seize voix contre quatre ${ }^{57}$. Leur solution, finalement reproduite dans le sénatus-consulte de 1865 , conditionne l'accession à la citoyenneté à l'expression d'une volonté libre en faveur de la cité française. C'est pourquoi ses partisans la qualifient de "libérale ${ }^{58}$. On peut pourtant se demander comment un individu dans le contexte de l'Algérie coloniale peut choisir de se soumettre au code civil. La question se pose particulièrement au regard de la situation juridique interstitielle qu'occupent les juifs algériens dans les années 1860 .

\section{LE STATUT PERSONNEL JUIF COMME «SCANDALE»}

L'application de la loi mosaïque aux contestations concernant la validité ou la nullité des mariages et répudiations avait été reconnue par l'ordonnance sur l'organisation de l'ordre judiciaire du 10 août 1834, et confirmée par l'ordonnance du 26 septembre 1842. Néanmoins les tribunaux rabbiniques sont éliminés en 1845 . Tribunaux et magistrats français ont dès lors la responsabilité de juger les affaires de mariage et d'héritage en appliquant la loi mosaïque même quand celle-ci s'oppose aux règles du code civil - y compris en matière de polygamie, de lévirat et de divorce. En 1807, le Grand Sanhédrin avait aboli ces "coutumes spéciales" pour les juifs français, mais ses décisions ne pouvaient être étendues aux juifs d'Algérie, puisqu’ils n'étaient pas français ${ }^{59}$.

Les usages des juifs algériens, maintenus dans leur «statut personnel» mosaïque, apparaissent aux juifs de la métropole gagnés à la cause du progrès comme une véritable anomalie nécessitant une "mission civilisatrice" à l'endroit de leurs coreligionnaires ${ }^{60}$. La réforme de l'organisation familiale à travers l'application du code civil est un élément essentiel de ce projet ${ }^{61}$. Ainsi, il n'est pas étonnant que les débats sur la nationalité des indigènes se soient

56. Séance du 9 octobre 1860, Conseil général d'Alger, Procès-verbaux, 1860, p. 115-6.

57. Séance du 9 octobre 1860, Conseil général d'Alger, Procès-verbaux, 1860, p. 123.

58. Séance du 9 octobre 1860, Conseil général d'Alger, Procès-verbaux, 1860, p. 121-22.

59. René Gutman, Les décisions doctrinales du Grand Sanhédrin, 1806-1807, Strasbourg, Presses universitaires de Strasbourg, 2000, p. 29-33.

60. Lisa Moses Leff, Sacred Bonds of Solidarity: The Rise of Jewish Internationalism in NineteenthCentury France, Stanford, Stanford University Press, 2006, p. 131; Joshua Schreier, 'Napoléon's Long Shadow', loc. cit.

61. Cf. par exemple l'article critiquant les rabbins algériens autorisant des mariages polygames et 
cristallisés sur des cas impliquant des juifs. Le premier jugement déclarant que les indigènes d'Algérie étaient des nationaux mais pas des citoyens français fut rendu au sujet d'un avocat juif algérien formé en France et réclamant le droit d'être admis au barreau d'Alger. La Cour de cassation finit par reconnaître ce droit à Elie-Léon Enos, mais établit aussi une distinction entre nationalité et citoyenneté, fondée sur des différences dans «la religion, les mœurs, la constitution du mariage, l'organisation de la famille». Comme le précise la décision de la cour d'Alger, "un grand nombre des droits que confere le statut personnel du musulman ou de l'indigène israélite ne sauraient se concilier avec les devoirs imposés aux citoyens français, dont il ne saurait secouer le joug sans contrevenir aux principes d'ordre public et mêmes aux lois pénales sous la double protection desquelles vit la nation française ${ }^{62}$. Ces références à l'ordre public et aux lois pénales renvoient implicitement aux «droits» des musulmans et des israélites à la polygamie, et la monogamie apparaît ici comme «le joug» de la citoyenneté. Qu'en est-il alors des indigènes adoptant le code civil «français»? Une multitude d'actions dans des affaires de divorce et d'héritage portées par des juifs algériens devant des tribunaux français après la disparition des tribunaux rabbiniques laissent à penser que la logique au fondement du statut juridique des indigènes s'impose avec difficulté. En effet, un justiciable avait toujours la faculté d'opter pour l'application du code civil. Si les tribunaux inférieurs, notamment le tribunal d'Alger, choisissaient souvent de suivre les lois françaises, la cour d'appel d'Alger, cependant, était plus circonspecte. Ses jugements faisant application de la loi mosaïque suivaient une double logique : la nécessité de protéger les coutumes indigènes mais aussi la "dignité» de la citoyenneté française qui impliquait de respecter les «bonnes mœurs" associées à l'ordre public ${ }^{63}$.

La confusion s'accroît avec la loi du 5 septembre 1851 qui facilite les mariages des israélites devant les officiers d'état civil français ${ }^{64}$. De tels mariages indiquaient-ils une intention d'être mariés selon la loi française plutôt que mosaïque? Quelle place faire à de telles manifestations d'intention dans le système du statut des personnes qui fait une large place, comme on l'a vu, à la notion de "volonté»? Allaient-elles déboucher sur la "naturalisation" collective réclamée par les réformateurs juifs? Ces questions sont vivement

le divorce (L'Univers israélite, $\mathrm{n}^{\circ} 10$, juin 1864, p. 184-185), ainsi que la défense de ces pratiques par le secrétaire du Consistoire d'Alger (L'Univers israélite, $\mathrm{n}^{\circ} 11$, juillet 1864, p. 532.

62. Enos, Cour d'Alger, 24 février 1862, in Robert Estoublon, Jurisprudence algérienne, tome 3, Alger, Adolphe Jourdan, 1862, p. 14-15. Sur le cas Enos comme pour le senatus-consulte de 1865, on se reportera à Mohamed Sahia Cherchari, «Indigènes et citoyens ou l'impossible universalisation du suffrage", Revue française de droit constitutionnel, $\mathrm{n}^{\circ}$ 60, 2004, p. 741-770.

63. Voir par exemple, Veuve Beneschti v. Beneschti, Tribunal d'Alger, 19 décembre 1845, in Robert Estoublon, Jurisprudence algérienne, tome 1, p. 22-23; Attali c. Attali, Cour d'Alger, 29 janvier 1857, in idem, tome 2, p. 6-9

64. Cf. "Décret qui affranchit des droits de timbres et d'enregistrement (5 septembre 1851)", in Robert Estoublon et Adolphe Lefébure, Code de l'Algérie, op. cit., p. 144. 
débattues pendant toute la décennie 1860 et perdurent même après la naturalisation collective des juifs en 1870 .

La jurisprudence souvent contradictoire sur ces questions laisse à voir une tension fondamentale entre la possibilité laissée aux individus de choisir le code civil et la cohérence des catégories de personnes au fondement de l'organisation judiciaire de l'Algérie. Les juifs algériens (et leurs avocats) exploitent les ambivalences et les contradictions de la loi. Vers la fin des années 1860, les juristes comme les journalistes en viennent à décrire les pratiques maritales des juifs algériens comme une menace grave à "l'ordre public». Si le code civil accordait des droits aux femmes, notamment en matière d'héritage, il était également invoqué par de nombreux hommes juifs en cas de divorce ${ }^{65}$. Un long procès opposa par exemple Guenouma Strock et Simon Courcheyia, la première arguant de l'impuissance du second. Or l'impuissance n'était plus alors considérée par le Code comme un motif d'annulation ${ }^{66}$. Le couple s'était marié devant l'officier d'état civil d'Oran en 1854. Quand Guenouma chercha à obtenir le divorce en application de la loi mosaïque, Simon réclama la protection du Code. Le tribunal d'Oran trancha en faveur de ce dernier, mais la cour d'Alger résista aux arguments assimilationnistes du tribunal de première instance, refusant de considérer que le couple avait renoncé à son statut personnel israélite en demandant à un officier d'état civil de prononcer leur mariage. La cour de cassation puis la cour d'Aix adoptèrent la position du tribunal d'Oran ${ }^{67}$. Pour ces deux juridictions, l'officier d'état civil incarne le Code. Le jugement final affirme que la "comparution libre et volontaire» du couple «devant un officier d'état civil ne peut s'expliquer que par la volonté de placer ce contrat sous la loi française». Plus profondément, le procès Courcheyia indiquait que «l'assimilation progressive des israélites indigènes avec les Français d'Europe est dans la pensée évidente du gouvernement français ${ }^{68}$.

Pourtant la cour d'Alger maintint sa position et continua de reconnaittre le statut personnel mosaïque, y compris le «droit» à la polygamie, comme le montre le procès de Judas Zermati en 1865. Jugée par le tribunal civil d'Alger en 1862, l'affaire opposait les enfants issus de son premier mariage à Zermati lui-même, sa seconde épouse et leurs enfants. Le premier mariage de Zermati avait été contracté sous la loi mosaïque avec Ricca bent Zermati

65. Cf. Aimé Poivre, Les indigènes algériens, leur état civil et leur condition juridique, Alger, Dubos, 1862, p. 25.

66. Cf. Dame Courcheyia c. Courcheyia, Cour d'Alger, 19 janvier 1860, in Robert Estoublon, Jurisprudence algérienne, tome 3, 1860, p. 1-4; Cour d'Alger, 18 mai 1860, ibidem, p. 27-29; Courcheyia c. Dame Courcheyia, Cour de cassation, 15 avril 1862, ibidem, p. 25-26. Sur l'Ancien Régime, Pierre Darmon, Le tribunal de l'impuissance : virilité et défaillances conjugales dans l'Ancien Régime, Paris, Éditions du Seuil, 1979.

67. Courcheyia c. Dame Courcheyia, Cour de cassation, 15 avril 1862, in Robert Estoublon, Jurisprudence..., op. cit., p. 25-26.

68. Dame Courcheyia c. Courcheyia, Cour d'Aix, 2 juin 1864, in Robert Estoublon, Jurisprudence..., op. cit., tome 3, 1864, p. 19-21 
en 1832. Son second mariage, avec Ricca Tabet, célébré en 1847 par un «rabbin de Jérusalem» itinérant "fut vu d'un très mauvais œil» par sa première femme et sa famille, qui «mirent tout en œuvre pour [le] détourner de contracter cette nouvelle union ${ }^{69}$. Les huit enfants issus du second mariage n'étaient inscrits aux registres de l'état civil que comme "enfants naturels». Quand, en 1862, Zermati s'adressa au tribunal d'Alger pour les faire reconnaître comme légitimes, les enfants issus de son premier mariage contestèrent vigoureusement la légalité de cette seconde union. Cette querelle de filiation et d'héritage prit une importance symbolique dans le contexte des débats sur le statut juridique des juifs algériens. Favorable aux arguments avancés par les enfants du premier mariage, le tribunal d'Alger trancha en leur faveur, et alla jusqu'à déclarer qu'il était $\mathrm{du}$ "devoir» des tribunaux français de réprimer "tout ce qui dans les mœurs et les usages [était] contraire à la morale et à l'ordre public ${ }^{70}$.

Quand l'appel de Zermati fut entendu par la cour d'Alger, l'avocat général Mazel hésita à défendre la légalité de la polygamie. Il affirma néanmoins qu' "au point de vue purement doctrinal», la bigamie était légale pour les juifs algériens ${ }^{71}$. Se rangeant à cet argument, la cour donna raison à Zermati, et expliqua que, jusqu'à ce qu'une nouvelle loi soit adoptée, les tribunaux étaient dans l'obligation "d'appliquer la loi mosaïque, de même que chaque jour, dans les instances entre musulmans, ils appliquent la loi mahométane, quelque tranchées que soient les différences entre ces lois et le droit civil français $»^{72}$.

Les partisans de l'assimilation israélite, au nombre desquels figuraient le consistoire d'Alger et des juristes comme Casimir Frégier, firent campagne pour "régulariser» cette situation scandaleuse ${ }^{73}$. Frégier, qui occupait le poste peu prestigieux de président du tribunal de première instance de Sétif, assura l'avancement de sa carrière en revendiquant l'organisation et la codification des lois algériennes ${ }^{74}$. Il insista sur les différences entre indigènes juifs et musulmans pour faire avancer la cause de l'accession des juifs à la citoyenneté, demandant par exemple : «Quoique pratiquant ou pouvant pratiquer la polygamie, ont-ils, comme les Musulmans d'Alger, par l'organe de leurs représentants au Conseil général, revendiqué le Droit au sérail? » ${ }^{75}$.

69. Zermati c. Zermati, Cour d'Alger (22 mai 1865), Journal de la jurisprudence de la Cour d'Alger [ci-après Robe], 1865 , p. 31 .

70. Jugement du tribunal d'Alger, 7 juillet 1864, cité dans Zermati c. Zermati, Cour d'Alger (22 mai 1865), in Robe, 1865, p. 35-36.

71. Plaidoyer du permier avocat général Mazel, cité dans Zermati c. Zermati, Cour d'Alger (22 mai 1865), Robe, 1865, p. 37. Cf. «La bigamie chez les Israélites algériens», L'Akhbar, 25 mai 1865.

72. Décision de la Cour d'Alger, "Zermati c. Zermati, Cour d'Alger (22 mai 1865) », Robe, 1865, p. 49.

73. Annie Rey-Goldzeiguer, Le royaume arabe, op. cit., p. 114.

74. Casimir Frégier, Du droit algérien, sa nature, ses éléments, son caractère : essai d'introduction à un cours de droit algérien, Alger, Dubos, 1861, p. 20; Casimir Frégier, De la législation algérienne, sa codification, sa révision, sa réformation, Sétif, Vincent, 1862.

75. Casimir Frégier, Juifs algériens : leur passé, leur présent, leur avenir juridique, leur naturalisation 
Frégier critiquait violemment la jurisprudence. C'était la définition même de la notion juridique de personne (et donc le domaine du droit personnel) qui était en jeu dans ces débats. La personne juridique - concept que Frégier distingue de "l'individualité" - ne saurait pour lui être l'objet d'un renoncement volontaire, y compris par la décision de se marier devant un officier d'état civil français ${ }^{76}$. "Vainement se récrierait-on contre l'immoralité, aux yeux de la loi française, des conséquences du mariage israélite telles que la polygamie, le divorce, etc.», affirmait-il. «Ces conséquences, la loi algérienne les a-t-elle admises pour l'Israélite et pour le Musulman? Si oui, (et comment répondrait-on négativement?) faites donc le procès à la loi ${ }^{77}$. C'est précisément ce à quoi il se consacre dans son long traité sur Les Juifs algériens.

\section{LE CHOIX IMPOSSIBLE DU SÉNATUS-CONSULTE}

Il y avait donc un consensus sur le fait que seule une solution législative parviendrait à régulariser la position ambiguë des juifs. Les partisans de l'assimilation avaient bon espoir que la réforme tant attendue soit décidée après la seconde visite de Napoléon III en Algérie, en 1865. Des rabbins s'adressèrent à l'empereur dans l'espoir qu'il intervienne pour corriger la situation : «le mariage, qui est à la base de la famille, est devenu une cause de perturbation et de scandale ${ }^{78}$. Le sénatus-consulte de 1865 tente de remédier, au moins partiellement, au problème.

Selon les propositions énoncées par les conseils départementaux, le décret met en place un processus de "naturalisation " pour les étrangers comme pour les musulmans et les juifs indigènes. Dans ces deux derniers cas, les individus doivent renoncer à la loi coranique, à la loi coutumière kabyle ou au statut personnel mosaïque afin de devenir citoyens, le conflit entre ces codes et la citoyenneté française portant atteinte à l'ordre public ${ }^{79}$. Le discours du sénateur Delangle insiste notamment sur le conflit entre la polygamie et le droit français. D'après lui, si du statut «qu'ils ont abandonné naissaient des droits et des usages incompatibles avec la pudeur publique, avec la morale, avec le bon ordre des familles, ces droits sont anéantis [...]. Il ne peut sur le sol de la patrie exister des droits contradictoires ${ }^{80}$. En décrivant la polyga-

collective, Paris, Michel Lévy, 1865, p. 351-2. Cf. Wilfred de Fonvielle, «Le Droit au sérail», Revue du monde colonial, Tome 7, n. 14, Janvier 1865, p. 64-69. Et le débat analysé dans Joshua Schreier, 'Napoléon's Long Shadow,' loc. cit

76. Casimir Frégier, Juifs algériens..., op. cit., p. 139-141.

77. Ibidem, 165 .

78. Casimir Frégier reproduit ces prières dans son texte, Idem., 446. Cf. «Adresse des Israélites d'Alger à sa Majesté Napoléon III", L'Akhbar 19 May 1865, p. 2

79. Voir «Exposé des motifs de M. Flandin, conseiller d'État, le 22 juin 1865 », in Robert Estoublon et Adolphe Lefébure, Code de l'Algérie, op. cit., p. 303. Cf. Michael Brett, 'Legislating for Inequality in Algérie,' loc. cit. et Schreier, 'Napoléon's Long Shadow,' loc. cit.

80. "Rapport présenté par M. Delangle le 30 juin 1865», in Robert Estoublon et Adolphe Lefébure, Code de l'Algérie, op. cit., p. 305. 
mie comme un "droit», Delangle place le statut juridique des indigènes en contradiction avec les droits des citoyens. Aux yeux de plusieurs sénateurs, cependant, la proposition est peu convaincante voire risible, si l'on se fie aux notations portées aux procès-verbaux. Quand Michel Chevalier propose l'abolition de la polygamie, le comte général de la Ruë répond que «si on leur propose de devenir citoyens français au prix de ce sacrifice, de ne pouvoir reprendre une jeune femme quand celle qu'ils ont est devenue vieille, ils ne seront sans doute pas très tentés d'user de ce droit à une telle condition $»^{81}$. Ces partisans de l'assimilation juridique de l'Algérie s'accordent ainsi pour dire que les indigènes choisiront la polygamie plutôt que la citoyenneté et le droit français.

Pour les colons, cependant, le véritable «scandale» est que les propriétés indigènes restent placées sous la «loi musulmane». Ainsi l'avocat Joseph Guérin écrit dans son journal l'Akhbar, en réponse à la proposition de Chevalier d'abolir la polygamie : "Nous n'en aurions pas tant demandé. Nous aurions souhaité qu'en devenant Français, les Musulmans, au lieu de demeurer soumis à la loi musulmane pure, devinssent dépendants de la loi française pour tout ce qui est placé en dehors du statut personnel. Ce progrès, plus modeste que celui que M. Michel Chevalier déclarait dès à présent réalisable, nous aurait paru suffisant, il aurait préparé l'autre progrès, plus complet, et par tant plus désirable, c'est-a-dire l'avènement du peuple arabe à la nationalité française ${ }^{82}$. Les colons veulent ainsi circonscrire la loi musulmane au statut personnel, pour que les biens fonciers soient placés sous le régime foncier français et puissent faire l'objet de libres transactions.

Malgré l'adoption du décret de 1865, les débats sur l'expansion du code civil aux indigènes algériens se poursuivent de plus belle. Alors que des juristes travaillent à formaliser le droit du statut personnel mosaïque, les colons et les partisans de l'assimilation des juifs continuent de réclamer l'extension du pouvoir civil et donc, avec lui, du code civil - même s'ils le font pour des raisons très différentes ${ }^{83}$. Certains critiques du sénatus-consulte de 1865 font de la polygamie un argument central. Au Sénat, les partisans de la colonisation libre dénoncent la loi comme incohérente et anticonstitutionnelle, notamment parce qu'elle permettait aux indigènes d'accéder à la fonction publique, civile et militaire, tout en leur refusant la pleine citoyenneté. Ils voient ce texte comme une nouvelle protection des "droits" des indigènes au détriment de ceux des colons. Le vicomte Victor-Ambroise Lanjuinais

81. Cf. «Délibération sur le projet de Sénatus-consulte relatif à l'état des personnes et à la naturalisation en Algérie», in Procès-verbaux des séances du sénat, tome 5, n 35-39, 1-7 juillet 1865, p. 229-230

82. Cf. Joseph Guérin, «Le sénatus-consulte I», L’Akbbar, 27 juillet 1865, p. 1. Ils proposent de faire du mariage une exception, $c f$. Clément Duvernois, "La question algérienne II", L'Akhbar, 14 décembre 1865 , p. 1

83. Cf. "Appel aux Israélites de l'Algérie, au sujet de leur naturalisation", et Arnold Thomson, "Des députations israélites se sont présentées à sa Majesté à chacun de ses voyages, pour demander la naturalisation ", L'Akbbar, 18 mai 1866. Sur le droit du statut personnel mosaïque, $c f$. Edouard Sautayra, Code rabbinique. Eben Haezer, Paris, Challamel, 1868. 
affirme ainsi que la loi crée des distinctions injustes entre Français : «Il y aurait, contrairement à toutes les notions de justice et de raison, des Français qui ne seraient pas soumis à la loi française, des Français qui ne seraient pas Français ». Comment des indigènes algériens possédant la nationalité française peuvent-ils jouir de droits dont sont exclus les Français soumis à la «loi française»? En France, explique-t-il, "l'adultère est puni; la loi musulmane le considère en certains cas comme un droit. Ainsi tout le monde sait que par la loi musulmane tout mahométan peut avoir plusieurs femmes légitimes et qu'il peut en outre faire habiter la maison conjugale par un nombre indéfini de concubines. Voilà la loi musulmane ${ }^{84}$. Retournant les arguments de Delangle pour critiquer le projet de loi, Lanjuinais affirme que tous ceux qui possèdent la «qualité de Français» devraient avoir l'obligation de se soumettre au code civil.

D'autres cependant avancent que la «polygamie» ne devrait pas faire obstacle à une assimilation juridique au moins partielle. Une opinion publiée dans L'Akhbar par son rédacteur Arnold Thomson fait remarquer que «l'obstacle que sans cesse on oppose, qui sert de bouclier, que présentent comme un éventail ceux qui ne veulent pas faire nôtres ceux de la religion musulmane, c'est la polygamie que la leur admet et que la nôtre répudie». Plutôt que de faire de cette différence un obstacle insurmontable, suggère-t-il, pourquoi " ne pas introduire dans notre loi une modération qui donnerait satisfaction aux mours, aux usages du peuple que nous voulons nous assimiler? ${ }^{85} \mathrm{Le}$ prétendu "droit» à la polygamie peut donc être interprété soit comme une violation scandaleuse des lois françaises ou bien comme une exception sans importance. Quelle que soit la position défendue, le recours à la polygamie est cependant utilisé pour critiquer le décret de 1865 comme un obstacle à la poursuite de la colonisation.

Les critiques des colons envers la politique du gouvernement deviennent encore plus vives à la suite de la famine de $1868^{86}$. Faisant de la crise une nouvelle occasion de dénoncer les bureaux arabes, les partisans du gouvernement civil affirment que les autorités militaires, en maintenant et même parfois en renforçant les coutumes et les lois primitives des populations indigènes, constituent le principal obstacle à l'assimilation. Les exhortations du directeur de L'Indépendant de Constantine, Jules Vinet, fournissent un exemple typique de ces critiques. Selon lui, pour parvenir à de véritables réformes, «il s'agit de placer les Arabes sous le code civil, en ne réservant que quelques questions spéciales, comme le divorce et la polygamie ${ }^{87}$, réclamant en

84. Annales du Sénat et du Corps législatif, tome 2, séance du 3 mars 1866, p. 93.

85. Arnold Thomson, "Assimilation (suite et fin)", L'Akhbar, 20 novembre 1866, p. 1

86. Sur la famine, $c f$. Bertrand Taithe, 'Algerian Orphans and Colonial Christianity in Algeria, 1866-1939', French History 20, n 3, 2006, p. 43 et surtout l'article qu'il lui consacre dans ce numéro.

87. Jules Vinet, Le droit commun pour les indigènes en Algérie, Paris, A. Le Chevalier, 1869, p. 7-8. 
d'autres termes, la création d'un «statut personnel musulman» entièrement détaché du droit foncier.

C'est également ce que propose François Cadoz, huissier à Mascara et expert autoproclamé en droit islamique dans son traité - explicitement favorable aux colons - sur Le droit musulman malékite. Rédigé après l'instauration du pouvoir civil en 1870, l'ouvrage propose un modèle de «statut personnel» musulman. L'auteur avance «que les principes de la loi musulmane et du dogme islamique bien compris et bien appliqués, ne s'opposent pas au développement civilisateur des peuples musulmans» et que "les mêmes principes, exceptés ceux qui ont trait au divorce et aux successions, ne s'opposent point à l'application du code civil français aux arabes de l'Algérie ${ }^{88}$. Par ailleurs, Cadoz est favorable à la naturalisation collective des juifs accordée par le décret Crémieux de 1870. Cette position se fonde sur des critères moraux de la population juive et notamment leurs valeurs familiales : «Les hommes sont laborieux, économes, bons pères, bons époux; les femmes sont vertueuses, elles excellent dans les soins domestiques; beaucoup d'entre elles, par leur éducation, leur talent, leur mise simple et élégante, peuvent rivaliser dans les salons avec les dames françaises ${ }^{89}$.

Promulgué lors d'une période de crise et de transition politique, le décret Crémieux abolit le «statut personnel» des juifs ${ }^{90}$. Un «statut personnel» musulman est en revanche institué peu de temps après, avec l'un des articles de la loi Warnier de 1873 qui réorganise la propriété foncière. Rendue possible par le passage d'un gouvernement militaire à un gouvernement civil en Algérie, la réforme du droit foncier élaborée par Auguste Warnier consolide cette nouvelle catégorie juridique en la détachant entièrement du statut réel des musulmans algériens. Il explique son intention d'étendre au territoire algérien le droit foncier français dans son rapport à l'Assemblée nationale : "Nous distinguons entre le statut réel et le statut personnel des musulmans de l'Algérie. - Nous respectons ce dernier, qui touche par divers points à la liberté de conscience, à la religion, à la vie intime de la famille; mais nous considérons comme un devoir de retenir le statut réel, celui qui touche aux intérêts immobiliers, pour le soumettre à la loi française, aux principes fondamentaux de notre droit public partout où flotte le drapeau national ${ }^{91}$. Ainsi, avec cette loi, les intérêts fonciers des colons coïncident avec les termes et la logique mis en place par le sénatus-consulte de 1865. Désormais circonscrit à un domaine du «statut personnel» indépendant du droit foncier, le droit musulman à la polygamie n'est plus conçu comme une institution

88. François Cadoz, Droit musulman Malékite, Paris, Challamel, 1871.

89. Ibidem, p. 195.

90. Décret du 24 octobre 1870, Bulletin officiel du gouvernement d'Algérie, 1871, p. 336.

91. Rapport fait par M. Warnier, membre de l'Assemblée nationale, «Loi relative à l'établissement et à la conservation de la propriété en Algérie", in Robert Estoublon et Adolphe Lefébure, Code de l'Algérie, op. cit., p. 399. Cf. Charles-Robert Ageron, Les Algériens musulmans et la France (1871-1919), tome 1, Paris, Presses universitaires de France, 1968, p. 78-83. 
économique susceptible de réforme, mais comme une institution religieuse et légale. La loi Warnier fonde sa légitimité sur cette prétendue protection des questions «intimes» relatives au statut personnel. L'article 7 déclare en effet qu' «il n'est point dérogé par la présente loi au statut personnel ni aux règles de succession des indigènes entre eux». Alors qu'elle promet de "protéger» ce statut personnel, cette loi l'institue de fait - et, avec lui, affirme un «droit à la polygamie». Ce droit symbolise dès lors l'insurmontable différence légale (et implicitement religieuse) du «musulman» - et donc son caractère non assimilable.

Dans les catégories du code civil, le statut personnel s'applique aux personnes, alors que le statut réel s'applique aux biens fonciers et aux choses. D'après les principes français du droit international privé, l'un est national, alors que l'autre est territorial. Leurs définitions respectives sont à la fois mutuellement constitutives et mutuellement exclusives ${ }^{92}$. Après 1865 , les indigènes algériens avaient officiellement la nationalité française; leur «statut personnel» avait donc un fondement incertain. Les juifs algériens qui demandèrent la protection du "droit français » incarnaient et exploitaient les conflits juridiques et politiques entre leur statut et leur nationalité. Les débats sur la loi mosaïque qui en résultèrent attirèrent l'attention des juristes sur la polygamie en tant que violation scandaleuse du code civil. A la suite du sénatus-consulte de 1865 , ils fournirent un vocabulaire et un cadre légal pour dénoncer la polygamie comme fondamentalement incompatible avec la citoyenneté française, et ce autant pour les musulmans que pour les juifs.

Les débats des législateurs sur la polygamie musulmane semblaient donc parallèles à ceux sur la polygamie juive. Il existait pourtant une différence cruciale dans l'apparente équivalence juridique entre le statut musulman et le statut personnel israélite : le droit foncier. Comme je l'ai suggéré ici, les débats sur la polygamie musulmane étaient historiquement liés à des débats sur la propriété et la colonisation, presqu'entièrement absents des discussions de la situation juridique des juifs algériens. Ainsi, même s'ils partageaient un même discours sur la polygamie en tant que scandale moral et légal, les officiers des bureaux arabes et les partisans des colons déployaient ce discours à des fins bien différentes de celles des partisans de l'assimilation des juifs.

Pour comprendre comment la polygamie en est venue à incarner l'incompatibilité du statut personnel musulman avec la citoyenneté française, il faut analyser les transformations qui ont eu lieu, non seulement dans les lois sur la citoyenneté et la nationalité des Algériens, mais aussi dans le droit

92. Maurice Block, Dictionnaire de l'administration française, Paris, Berger-Levrault, 1856, article «Statut», p. 1488. 
foncier. Nous avons vu que, dans les années 1850 et 1860, les partisans des colons affirmaient qu'une transformation du droit foncier mènerait à une réforme morale et sociale des indigènes algériens, à la disparition de la polygamie voire à une fusion des populations. Ces arguments envisageaient la polygamie comme une institution avant tout économique que la colonisation pourrait réformer, et non à travers le prisme de la religion. Certains partisans des colons étaient même prêts à accorder au mariage musulman un statut spécial en contrepartie d'une application du droit français comme "droit commun». Pourtant, après l'extension des règles du droit foncier aux propriétés musulmanes en 1873 , l'intérêt des colons pour l'assimilation des musulmans disparut presque entièrement ${ }^{93}$. C'est plutôt le maintien, par des moyens politiques, de limites à l'entrée dans la cité française et notamment de la condition du respect du Code qui devint pour eux capitale. Dès lors, le statut personnel musulman, récemment défini, vint à représenter un scandaleux mais nécessaire "état d'exception» qui confirmait à la fois la souveraineté juridique française et la dignité du titre de citoyen français.

Les implications de cette logique devinrent particulièrement tangibles quand, à partir de la fin des années 1880 , plusieurs députés et sénateurs se prononcèrent en faveur de l'extension des droits politiques aux sujets "musulmans», tout en préservant leur statut personnel (y compris le droit à la polygamie). Dans un contexte où l'on avance une assimilation politique (et non plus juridique), les élites politiques de la métropole affirment à leur tour que les différences légales sur le mariage, y compris la polygamie, ne constituaient qu'un détail mineur. Comme l'explique le sénateur de Martinique Pierre Isaac dans sa proposition de 1893, «il ne serait pas impossible, après avoir mis à part les prescriptions religieuses, maintenues strictement dans le domaine du statut personnel, de dégager, comme on l'a fait ailleurs, une législation locale qui ne présenterait, par rapport à la législation métropolitaine, que des différences de détail justifiées par la diversité des besoins». Il faisait remarquer que la plupart des aspects du droit français s'appliquent déjà aux indigènes algériens. D’après lui, ce serait "une erreur de dire que les musulmans d'Algérie ne sont pas soumis à la loi française». Leur statut juridique ne serait pas plus exceptionnel que celui des colons eux-mêmes, qui, après tout, bénéficient aussi de dispenses légales ${ }^{94}$.

Les juristes, au contraire, sceptiques face à ces propositions parlementaires déclarèrent que le «statut personnel» musulman était entièrement incompatible avec la citoyenneté française. Souvent, la «polygamie» incarnait cette différence scandaleuse et insurmontable. Dans une thèse de droit soutenue

93. Sur l'histoire de la législation foncière en Algérie, $c f$. Jean Louis Autin, «La législation foncière», loc. cit.; Alain Sainte-Marie, "Législation foncière et société rurale : L’application de la loi du 26 juillet 1873 dans les douars de l'Algérois", Etudes rurales, n 57,1975 , p. 61-87.

94. Proposition de loi par M. Pierre Isaac, Journal officiel. Documents parlementaires, Sénat, Annexe $\mathrm{n}^{\circ} 134,4$ avril 1893, p. 287. Il tire ses exemples des «vieilles colonies» et des établissements français de l'Inde. 
en 1894 et couronnée d'un prix, Emmanuel Besson soutient que ni les juristes, ni les moralistes ne peuvent tolérer les compromis alors évoqués au Parlement : "Il ne doit pas y avoir sur le sol français des citoyens ayant des droits contradictoires. Nous ne pouvons nous faire à l'idée qu'il fût permis à un Français, par cela seul qu'il serait de race arabe ou kabyle, d'épouser légalement quatre femmes ou de vendre sa fille impubère. Ce serait vraiment avilir le titre de citoyen français ${ }^{95}$. L'honneur national français, affirme-t-il, est lié à la supposée pureté (sexuelle) du droit. La nécessité politique de cette pureté condamnait le "musulman" à son état d'exception. Pour ces juristes, le droit français (et de ce fait la citoyenneté) et le droit "personnel» musulman étaient entièrement incompatibles. Et la plupart des colons avaient alors intérêt à préserver cette incompatibilité.

Judith Surkis est membre de la School of Social Science (Princeton), Institute for Advanced Study. Article traduite de l'anglais par François Proulx

95. Emmanuel Besson, La législation civile de l'Algérie. Étude sur la condition des personnes et le régime des biens en Algérie, Paris, Marescq, 1894, p. 340. 